\title{
Splanchnic Vasoregulation After Major Abdominal Surgery in Pigs
}

\author{
Lukas E. Brügger · Guido Beldi • Mario Beck • \\ Francesca Porta - Hendrik Bracht · Daniel Candinas • \\ Jukka Takala $\cdot$ Stephan M. Jakob
}

Published online: 10 April 2010

(C) Société Internationale de Chirurgie 2010

\begin{abstract}
Background Unrecognized reduction of blood supply to intestinal organs is associated with significant postoperative morbidity in abdominal surgery. The aim of this study was to determine whether-in the absence of hypovolemia-intestinal hypoperfusion as a result of blood flow redistribution occurs after abdominal surgery.

Methods Standardized operative trauma was induced in 14 healthy pigs. Systemic, regional, and local blood flow, intestinal and gastric intraluminal-to-end-tidal $\mathrm{pCO}_{2}$ gradients representing mucosal perfusion, and oxygen transport variables were measured for 10 postoperative hours. Normovolemia was maintained using continuous infusion of Ringer's lactate and additional boluses of colloids in response to blood pressure, pulmonary wedge pressure, and urinary output.

Results Postoperative blood flow was significantly increased in the celiac trunk (76\% increase [percentage of baseline flow], $p=0.003)$ and the hepatic (136\% increase, $p=0.002)$ and splenic (36\% increase, $p=0.025$ ) arteries. Blood flow was significantly decreased in the mesenteric artery (25\% decrease, $p=0.007)$ and portal vein $(13 \%$ decrease, $p=0.028$ ). Carotid and renal artery blood flow remained unchanged.

Conclusions Maintenance of normovolemia is insufficient to protect from intestinal hypoperfusion after abdominal surgery. Postoperative redistribution of cardiac
\end{abstract}

L. E. Brügger · G. Beldi · D. Candinas

Department of Visceral Surgery and Medicine, Bern University

Hospital and University of Bern, 3010 Bern, Switzerland

M. Beck · F. Porta · H. Bracht · J. Takala · S. M. Jakob $(\square)$

Department of Intensive Care Medicine, Bern University

Hospital and University of Bern (Inselspital),

3010 Bern, Switzerland

e-mail: stephan.jakob@insel.ch output results in decreased intestinal and increased hepatic and splenic arterial blood flow.

\section{Introduction}

Morbidity after routine, major, elective surgery remains high and occurs in up to $50 \%$ of patients [1-4]. Reduced gut mucosal perfusion is a risk factor for postoperative complications which potentially mediates distant organ injury. Perceived mechanisms include exacerbation of systemic inflammation in response to ischemia/reperfusion injury as well as translocation of bacteria with subsequent endotoxemia [5-7]. As demonstrated in a number of clinical studies, mucosal hypoperfusion is associated with prolonged stay in the intensive care unit, high morbidity, and increased costs [8-11]. During hypovolemia, intestinal blood perfusion is compromised earlier than the perfusion of other vascular beds [12]. Such intestinal vasoconstriction possibly occurs in order to maintain perfusion of other vital organs such as the brain and thoracic organs in hypovolemia, as demonstrated in experimental animal [13, 14] and human [15] studies.

During the present study, we tested whether vasoregulatory responses to major abdominal surgery result in relative intestinal hypoperfusion despite normovolemia. Such an observation would at least in part explain the remarkable morbidity rate in non-high-risk abdominal surgery. Understanding the mechanisms then would allow introduction of new strategies to reduce the postoperative complications.

\section{Materials and methods}

The study was performed in accordance with the National Institutes of Health guidelines for the care and 
use of experimental animals and with the approval of the Animal Care Committee of the Canton of Bern, Switzerland.

Anesthesia, premedication, and ventilation were performed as described in detail previously [16]. Briefly, 14 pigs $(37-43 \mathrm{~kg}$ ) were fasted overnight, premedicated with a combination of ketamine and azaperon, and given midazolam and atropine intravenously for endotracheal intubation. One dose of $1.5 \mathrm{~g}$ ceforoximum (Zinacef, GlaxoSmithKline AG, Münchenbuchsee, Switzerland) was given intravenously. Anesthesia was maintained with pentobarbital and fentanyl. Pancuronium $\left(1 \mathrm{mg} \mathrm{kg}^{-1} \mathrm{~h}^{-1}\right)$ was used as a muscle relaxant. The animals were ventilated with a volume-controlled mode.

A carotid arterial catheter and a pulmonary artery catheter were inserted via the left internal jugular vein. A second Swan-Ganz catheter was inserted into a liver vein through the right internal jugular vein. Afterwards the position was confirmed using ultrasonic imaging.

Ringer's lactate solution was given at $10 \mathrm{ml} \mathrm{kg}^{-1} \mathrm{~h}^{-1}$. Additional fluids were administered intravenously as a bolus of 50 or $100 \mathrm{ml}$ solution of hydroxyethyl starch (HES) 130/0.4 (Voluven $^{\circledR}$, Fresenius Kabi AG, Stans, Switzerland) if urinary output was below $0.5 \mathrm{ml} \mathrm{kg}^{-1} \mathrm{~h}^{-1}$ and/or mean arterial pressure (MAP) was less than $60 \mathrm{mmHg}$ and/or pulmonary wedge pressure was less than $5 \mathrm{mmHg}$.

\section{Surgery}

The abdominal cavity was exposed by a midline abdominal incision. A drainage catheter was inserted into the urinary bladder. Air tonometers (Tonometrics ${ }^{\circledR}$, Datex-Engström Tonometrics Division, Helsinki, Finland) were inserted into the stomach and jejunum after enterotomy in order to measure intraluminal-to-end-tidal $\mathrm{pCO}_{2}$ gradients. Coin-shaped Oxyflo laser Doppler probes (Oxford Optronix ${ }^{\circledR}$ Ltd., Oxford, UK) were surgically fixed to the gastric and ileomucosal surfaces to measure local (microcirculatory) flows. Needle-shaped Oxyflo laser Doppler probes were inserted into the liver and kidney parenchyma. A femoral vein catheter was inserted for fluid administration and to obtain blood samples.

For regional flow measurement, carotid, superior mesenteric, hepatic, splenic, and right kidney arteries, celiac trunk, and portal vein were exposed, and ultrasound transit time flow probes (Transonic ${ }^{\circledR}$ Systems Inc., Ithaca, NY, USA) were placed around the vessels after in vitro calibration.

After completion of all surgical procedures, the abdominal wall was approximated by clamps, and towels were placed over the wound to minimize heat loss.
Experimental protocol

The animals were observed for $10 \mathrm{~h}$ after surgery. After surgery, no further manipulations were permitted, except for a recruitment maneuver to keep the lungs open after $6 \mathrm{~h}$ of stabilization, preceded by a fluid challenge. Eight of these animals were also part of a study that started after the end of this experiment. In an experimental sepsis model, the effect of fluid resuscitation on mortality and organ function was evaluated [17].

Hemodynamic monitoring and data recording have also been described previously [16]. Briefly, heart rate, ECG, carotid and pulmonary artery occlusion pressure, and regional (ultrasound transit time flow probes) and microcirculatory (laser Doppler flow probes) blood flows were recorded continuously and visualized on computer monitors.

Blood samples for the measurement of hemoglobin, lactate, and blood gases (ABL 520 and OSM3, Radiometer, Copenhagen, Denmark) were taken at baseline and after $10 \mathrm{~h}$ from the carotid artery and portal/hepatic veins.

\section{Data analysis}

Hourly sets of 2-min median values of all variables were used for further analysis. No manipulations were allowed in the 15 min preceding each measurement. If predefined artifacts were detected within the 2-min measurement period, the period was shifted $10 \mathrm{~min}$ or less forward or backward. If this was not possible, the means of the preceding and subsequent values were used to have a full set of data for statistical analysis. If more than one value was missing, the data of this animal had to be excluded. Baseline was defined as the first full hour after animal preparation.

Systemic oxygen consumption, hepatosplanchnic oxygen consumption and extraction ratio, and hepatic lactate extraction ratio (baseline and end of experiment in 7 animals) were computed according to the formulas published previously [16].

Carbon dioxide gradients were calculated as the difference between tonometric and end-tidal $\mathrm{PCO}_{2}(\mathrm{kPa})$, which corresponds to arterial $\mathrm{PCO}_{2}$ [18] as long as cardiac output did not decrease to very low levels.

\section{Statistics}

For statistical analysis the SPSS software package, version 12.0 (SPSS, Inc., Chicago, IL, USA), was used. Since the variables were not normally distributed, nonparametric tests were used. Results are given as median (interquartile range) or percentages of baseline. Changes over time were assessed by the Friedman test. To characterize the time course of significant changes, the most aberrant value was 
compared to baseline using a Wilcoxon signed-ranks test. Statistical significance was considered to be $p \leq 0.05$.

\section{Results}

The median time of surgery was 360 (range $=318-400$ ) min. A median (interquartile range) of $650(513-1588) \mathrm{ml}$ of colloids was administered in addition to baseline crystalloids during the operation and postoperative stabilization. The intraoperative course of individual systemic hemodynamic parameters of the last $3 \mathrm{~h}$ is indicated in Fig. 1. Mean arterial pressure and cardiac index decreased

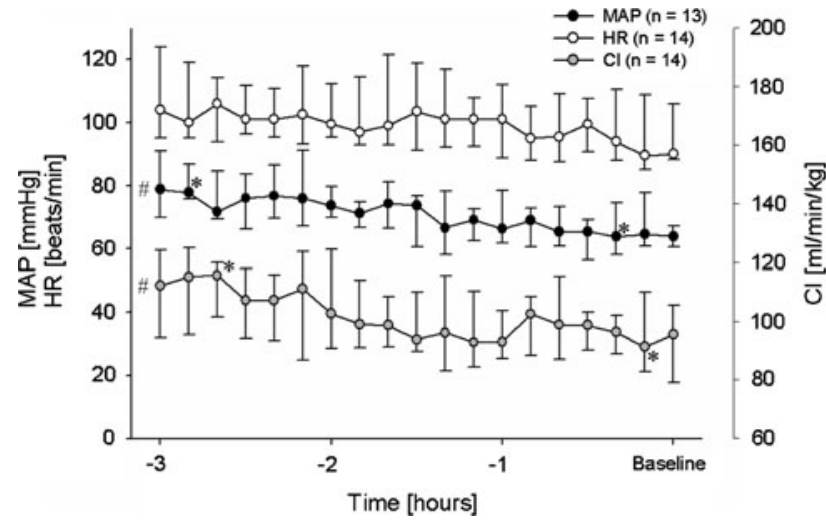

Fig. 1 Mean arterial pressure (MAP), heart rate (HR), and cardiac index $(\mathrm{CI}=$ cardiac output/body weight $)$ during the last $3 \mathrm{~h}$ of abdominal surgery. Values are sets of 2-min median values representative of intervals of $10 \mathrm{~min}$ and bars indicate interquartile range. ${ }^{\#} p<0.05$ for time effect (Friedman test); $* p<0.05$ for comparison between most different values (Wilcoxon signed-ranks test)

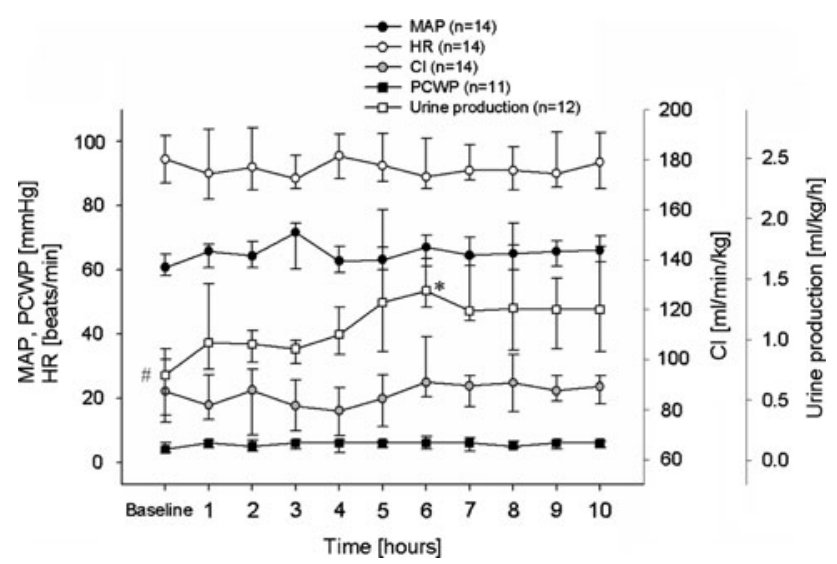

Fig. 2 Mean arterial pressure (MAP), heart rate (HR), cardiac index $(\mathrm{CI}=$ cardiac output/body weight $)$, pulmonary capillary wedge pressure (PCWP), and urine production during the postoperative period. Values are hourly sets of 2-min median values and bars indicate interquartile range. ${ }^{\#} p<0.05$ for time effect (Friedman test); $* p<0.05$ for peak value in comparison with baseline value (Wilcoxon signed-ranks test) slightly within normal ranges, while the heart rate remained stable. Postoperative systemic hemodynamic variables are shown in Fig. 2. Mean arterial pressure, heart rate, cardiac index, and pulmonary artery occlusion pressure remained stable during the postoperative period. Diuresis increased from $0.7(0.4-0.9)$ to $1.4(1.3-1.7)$ $\mathrm{ml} \mathrm{kg}{ }^{-1} \mathrm{~h}^{-1}(p=0.015)$.

Regional blood flows are shown in Fig. 3a, b. The flow in the superior mesenteric artery was significantly reduced at early time points. Conversely, celiac trunk flow progressively increased during the postoperative period. When flows are displayed as a fraction of cardiac output, reciprocal regulation of blood flow between the superior mesenteric artery and celiac trunk is evident (Fig. 4). Peak flows compared to baseline flows are shown in Fig. 5a, b. Carotid artery $\left(\right.$ median $=4.6 \mathrm{ml} \mathrm{min}{ }^{-1} \mathrm{~kg}^{-1}$, range $=4.3-5.3 \mathrm{ml} \mathrm{min}^{-1}$
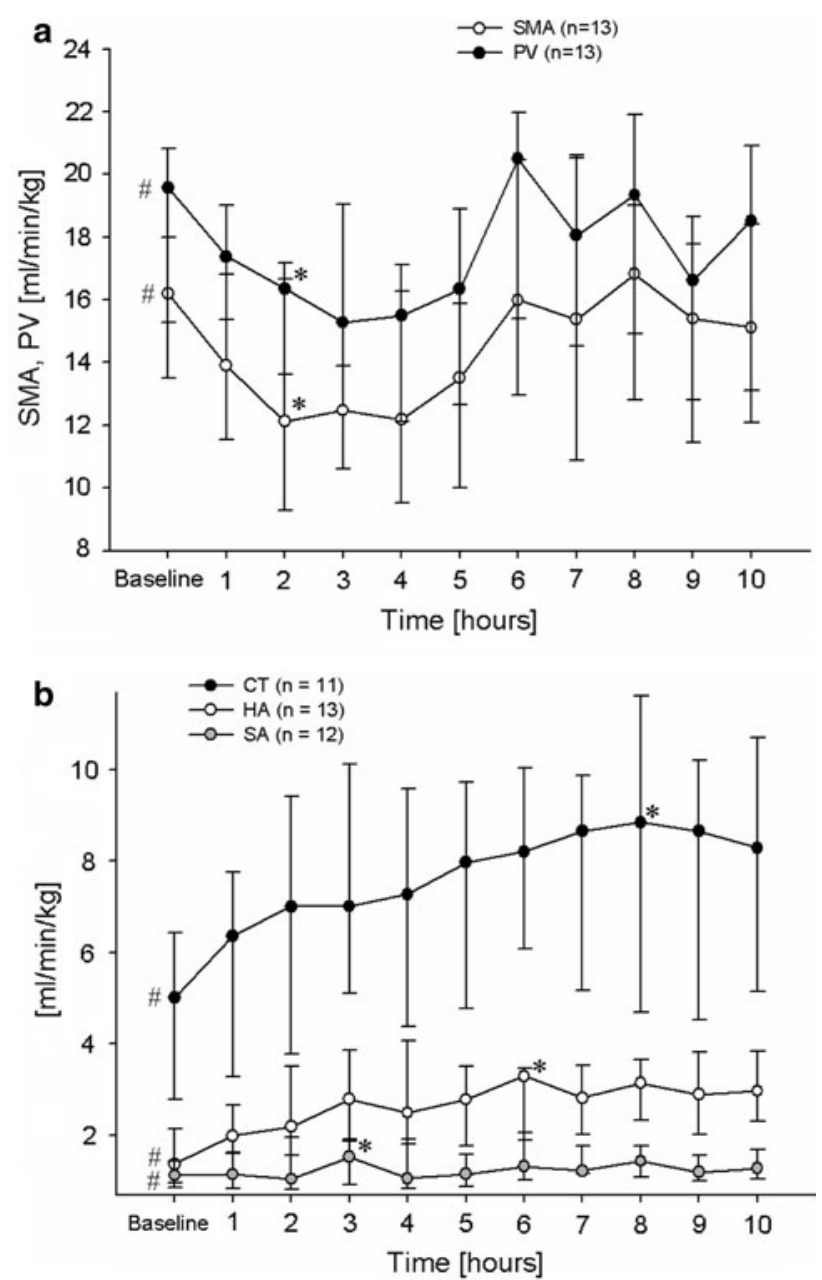

Fig. 3 a, b Regional flows of superior mesenteric artery (SMA), portal vein (PV), celiac trunk (CT), hepatic artery (HA), and splenic artery (SA). Values are hourly sets of 2-min median values and bars indicate interquartile range. ${ }^{\#} p<0.05$ for time effect (Friedman test); $* p<0.05$ for peak value in comparison with baseline value (Wilcoxon signed-ranks test) 


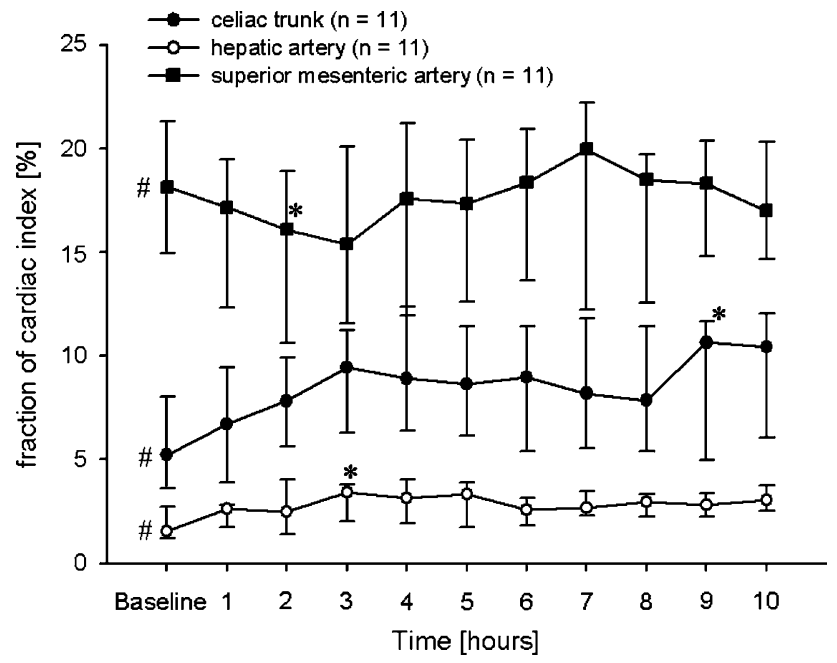

Fig. 4 Summary of regional flows of celiac trunk (CT), hepatic artery (HA), and superior mesenteric artery (SMA) as a fraction of cardiac index in the postoperative period. Values are hourly sets of 2-min median values and bars indicate interquartile range. ${ }^{\#} p<0.05$ for time effect (Friedman test); $* p<0.05$ for peak value in comparison with baseline value (Wilcoxon signed-ranks test)

$\mathrm{kg}^{-1}$ ) and renal artery (median $=4.4 \mathrm{ml} \mathrm{min}{ }^{-1} \mathrm{~kg}^{-1}$, range $=3.9-4.9 \mathrm{ml} \mathrm{min}^{-1} \mathrm{~kg}^{-1}$ ) flows remained stable during the experiment (data not shown).

Mucosal $\mathrm{pCO}_{2}$ values ( $\left.\mathrm{PrCO} 2\right)$ over time are displayed in Fig. 6. Mucosal end-tidal $\mathrm{pCO}_{2}$ gradients remained high (cutoff for postoperative functional recovery delay: $2.8 \mathrm{kPa}$ ) [11] without significant changes over time in the stomach $($ median $=2.8$, range $=1.8-3.7 \mathrm{kPa})$ and jejunum $($ median $=3.0$, range $=2.6-3.5 \mathrm{kPa}$ ).

Oxygen transport variables, arterial lactate extraction, and hepatic lactate extraction are given in Table 1. Systemic oxygen consumption decreased slightly, and hepatosplanchnic oxygen consumption and hepatic lactate extraction ratio remained stable. Hepatosplanchnic oxygen extraction (fraction of consumption from delivery) remained high throughout the observation period.

Microcirculatory flows (Fig. 7) were heterogeneous during the observation period, without significant changes over time.

\section{Discussion}

This is the first trial that describes blood flow distribution between abdominal organs up to $10 \mathrm{~h}$ after standardized abdominal surgery. The results of the study show that abdominal arterial blood flow was redistributed away from the gut to celiac trunk-supplied organs such as the liver and the spleen despite stable systemic hemodynamics and preserved urinary output. During the same period, nonsplanchnic blood flows remained unchanged. We therefore

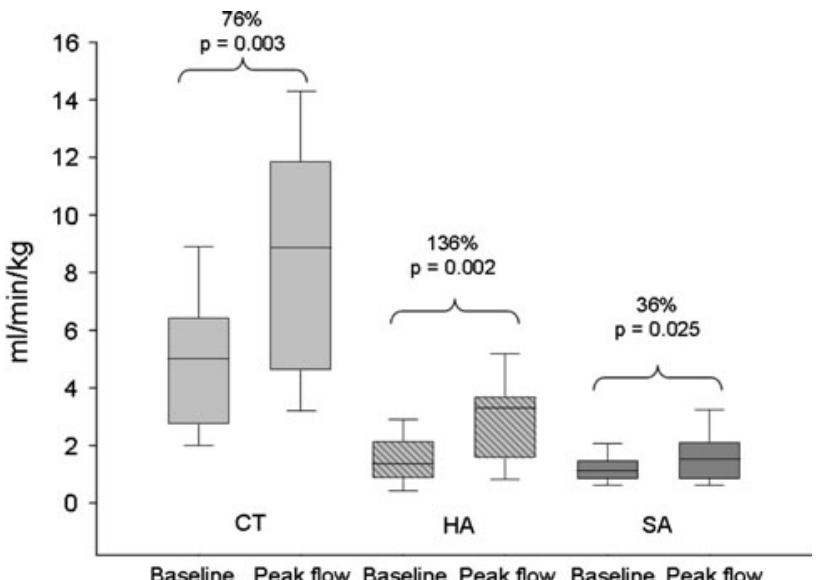

Baseline Peak flow Baseline Peak flow Baseline Peak flow

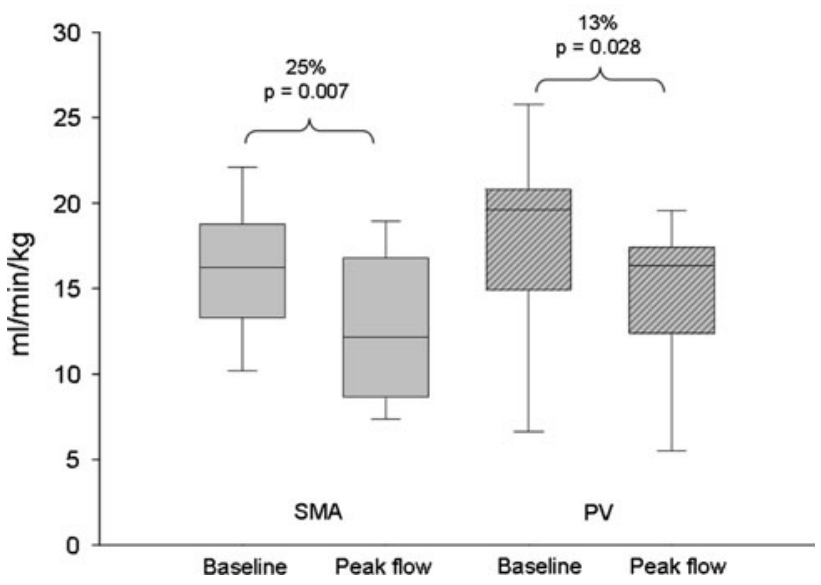

Fig. 5 Positive or negative peak flows of celiac trunk (CT), hepatic artery (HA), splenic artery (SA), superior mesenteric artery (SMA), and portal vein (PV) compared to baseline (box plots). The boundary of the box closest to zero indicates the 25th percentile, a line within the box marks the median, and the boundary of the box farthest from zero indicates the 75 th percentile. Whiskers (error bars) above and below the box indicate the 95th and 5th percentiles. Percentage values above the box plots indicate relative difference of the medians

postulate that vasoregulatory mechanisms protect the hepatic arterial blood supply at the expense of mesenteric perfusion in the postoperative period.

Reactive hypoperfusion in the mesenteric vascular bed had been described previously in short-term studies with other experimental setups such as hemorrhagic [13, 14], cardiogenic [19], or septic [20] shock. Similarly, in clinical studies, a parallel decrease of cardiac output and total hepatosplanchnic blood flow had been reported during hemorrhage [21], simulated hypovolemia [15], or hemodiafiltration [22]. Contrary to our experiment, all those studies were associated with hypovolemia and/or reduced cardiac output. However, when hypovolemia was combined with somatic afferent nerve stimulation simulating surgical trauma, blood flow reduction to the mesenteric region was even increased [23, 24]. In order to focus on sequelae of isolated surgical trauma, we excluded 


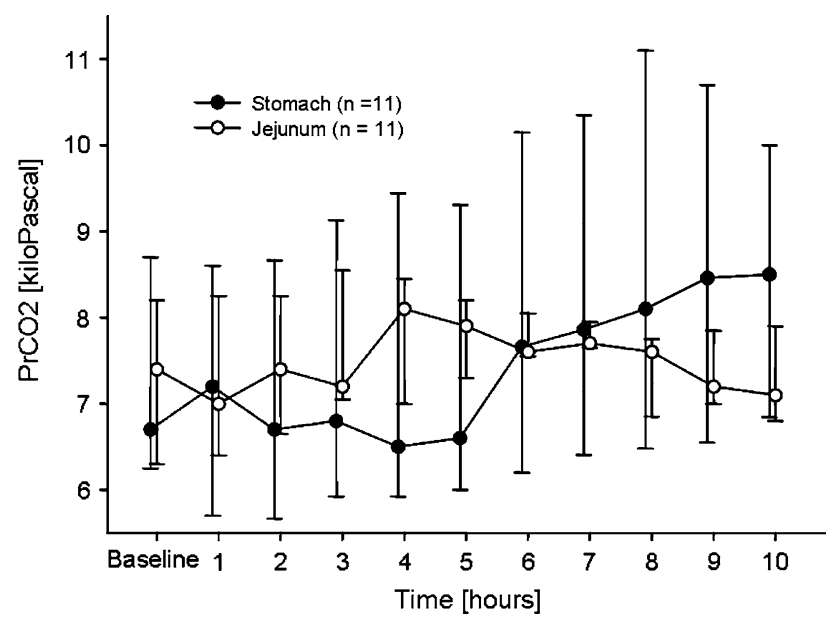

Fig. 6 Summary of mucosal $\mathrm{pCO}_{2}(\mathrm{PrCO} 2)$ of stomach and jejunum. Values are hourly sets of 2-min median values and bars indicate interquartile range. Time effect did not reach significance level (Friedman test)

hemorrhage and septic shock and maintained normovolemia throughout the experiment.

Jejunal mucosal $\mathrm{pCO}_{2}$ tended to increase within the first $5 \mathrm{~h}$, while gastric mucosal $\mathrm{pCO}_{2}$ did not. Accordingly, the early changes are consistent with the respective regional blood flow changes. Of note, mucosal $\mathrm{pCO}_{2}$ values are also dependent on concomitant changes in metabolism, hemoglobin, and $\mathrm{pH}$ [25]. Accordingly, the measured changes in $\mathrm{pCO}_{2}$ may not be the result of local blood flow changes alone.

The redistribution within splanchnic arteries and the subsequent decrease in intestinal perfusion are of potential clinical significance. Hepatosplanchnic oxygen extraction and mucosal end-tidal $\mathrm{pCO}_{2}$ gradients remained high throughout the study period, representing marginal mucosal perfusion. However, other indicators of inadequate splanchnic blood supply such as hepatosplanchnic oxygen consumption, hepatic lactate extraction, and systemic lactate concentrations remained normal.

The postoperative increase in celiac trunk and hepatic arterial blood flows may represent recovery from arterial vasospasms induced by the operative trauma. The so-called hepatic arterial buffer response is initiated by reduced flow of the superior mesenteric artery or portal vasculature, which then leads to compensatory hepatic arterial dilatation
[26]. Functional consequences of short-term blood flow redistribution toward the celiac trunk-perfused organs at the expense of the gut remain to be determined. Potentially, blood flow redistribution is additionally associated with increased perioperative hepatic metabolism.

Microcirculatory blood flows in all measured splanchnic organs were subject to great variability and did not show significant changes. Only in the kidney did constant microcirculatory perfusion seem to be the result of unchanged regional flow. Conversely, in the liver, stomach, and jejunum, regional flows were heterogeneous, possibly in response to flow redistribution within the organ, as described previously [27].

Various strategies that address cardiac output, such as fluid restriction [28-30] and liberal fluid administration $[31,32]$, have been investigated intensively in order to optimize postoperative outcome. A number of randomized studies have shown that maximizing stroke volume and oxygen delivery by volume loading to supranormal values may improve outcome during high-risk surgery and trauma associated with hypovolemia (goal-directed fluid management) [33-36]. These results potentially represent an effect of restoration of impaired intestinal perfusion. However, in order to prove such a hypothesis, postoperative intestinal perfusion has to be assessed as proposed in the present study. According to our study, volume substitution to supranormal levels could be justified in abdominal surgery, even in the absence of hypovolemia.

\section{Conclusions}

In this animal model we show postoperative blood flow distribution away from the superior mesenteric artery toward organs supplied by the celiac trunk. Such redistribution is associated with signs of marginal mucosal perfusion after abdominal surgery in the presence of normovolemia. The resulting blood flow reduction of the mesenteric region, which is particularly susceptible to hypoperfusion, may explain high morbidity after major abdominal surgery. Future studies investigating perioperative fluid management ideally would aim at monitoring and improving such postoperative blood flow redistributions.

Table 1 Summary of oxygen transport data for arterial lactate and hepatic lactate extraction in the postoperative period $(n=7)$

\begin{tabular}{|c|c|c|c|}
\hline & Baseline & $10 \mathrm{~h}$ & $p$ \\
\hline Systemic oxygen consumption $\left(\mathrm{ml} \mathrm{min}{ }^{-1} \mathrm{~kg}^{-1}\right)$ & $5.68(5.48-5.93)$ & $5.09(4.94-5.49)$ & 0.018 \\
\hline Hepatosplanchnic oxygen consumption $\left(\mathrm{ml} \mathrm{min}{ }^{-1} \mathrm{~kg}^{-1}\right)$ & $1.64(1.51-1.80)$ & $1.61(1.53-1.89)$ & 0.352 \\
\hline Hepatosplanchnic oxygen extraction ratio (\%) & $82(72-83)$ & $65(62-77)$ & 0.128 \\
\hline Arterial lactate $\left(\mathrm{mmol} \mathrm{l}^{-1}\right)$ & $1.3(1.2-1.5)$ & $0.7(0.6-0.8)$ & 0.018 \\
\hline Hepatic lactate extraction ratio $(\%)$ & $41(25-46)$ & $49(47-60)$ & 0.176 \\
\hline
\end{tabular}




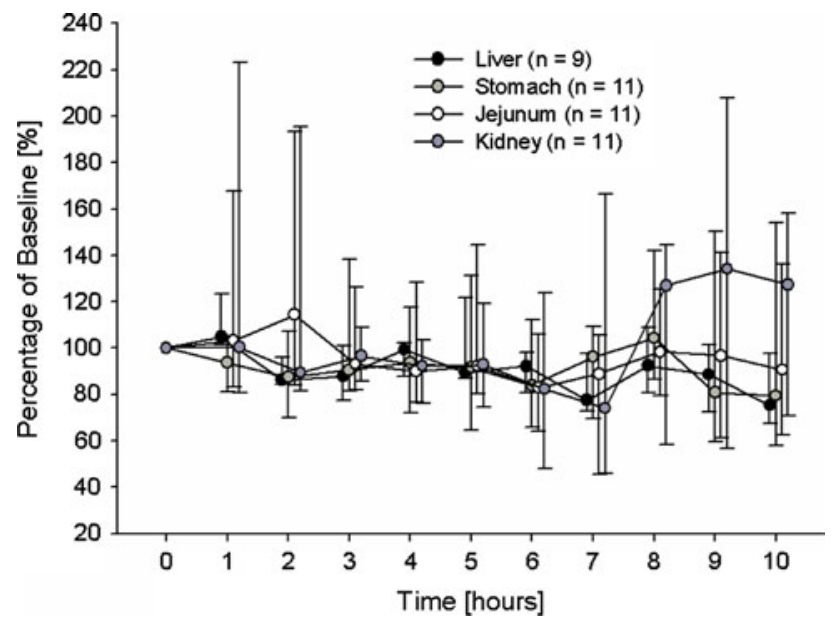

Fig. 7 Summary of microcirculatory flows within liver, kidney, and mucosa of stomach and jejunum. Values are hourly sets of 2-min median values and bars indicate interquartile range. No significant changes over time (Friedman test) were detected

Acknowledgment This work was funded by the Swiss National Fund for Scientific Research (SNF 3200BO-102268).

Conflicts of interest None.

\section{References}

1. Beldi G, Bisch-Knaden S, Banz V et al (2009) Impact of intraoperative behavior on surgical site infections. Am J Surg 198:157-162

2. Mazeh H, Samet Y, Abu-Wasel B et al (2009) Application of a novel severity grading system for surgical complications after colorectal resection. J Am Coll Surg 208:355-361

3. Luna A, Rebasa P, Navarro S et al (2009) An evaluation of morbidity and mortality in oncologic gastric surgery with the application of POSSUM, P-POSSUM, and O-POSSUM. World J Surg 33:1889-1894

4. Teh SH, Diggs BS, Deveney CW et al (2009) Patient and hospital characteristics on the variance of perioperative outcomes for pancreatic resection in the United States: a plea for outcomebased and not volume-based referral guidelines. Arch Surg 144:713-721

5. Baue AE (1993) The role of the gut in the development of multiple organ dysfunction in cardiothoracic patients. Ann Thorac Surg 55:822-829

6. Soong CV, Halliday MI, Barclay GR et al (1997) Intramucosal acidosis and systemic host responses in abdominal aortic aneurysm surgery. Crit Care Med 25:1472-1479

7. Kuzu MA, Tanik A, Kale IT et al (2000) Effect of ischemia/ reperfusion as a systemic phenomenon on anastomotic healing in the left colon. World J Surg 24:990-994

8. Mythen MG, Webb AR (1994) Intra-operative gut mucosal hypoperfusion is associated with increased post-operative complications and cost. Intensive Care Med 20:99-104

9. Bennett-Guerrero E, Panah MH, Bodian CA et al (2000) Automated detection of gastric luminal partial pressure of carbon dioxide during cardiovascular surgery using the Tonocap. Anesthesiology 92:38-45
10. Theodoropoulos G, Lloyd LR, Cousins G et al (2001) Intraoperative and early postoperative gastric intramucosal $\mathrm{pH}$ predicts morbidity and mortality after major abdominal surgery. Am Surg 67:303-308

11. Lebuffe G, Vallet B, Takala J et al (2004) A European, multicenter, observational study to assess the value of gastric-to-end tidal $\mathrm{PCO}_{2}$ difference in predicting postoperative complications. Anesth Analg 99:166-172

12. Hamilton-Davies C, Mythen MG, Salmon JB et al (1997) Comparison of commonly used clinical indicators of hypovolaemia with gastrointestinal tonometry. Intensive Care Med 23:276-281

13. Vatner SF (1974) Effects of hemorrhage on regional blood flow distribution in dogs and primates. J Clin Invest 54:225-235

14. Toung T, Reilly PM, Fuh KC et al (2000) Mesenteric vasoconstriction in response to hemorrhagic shock. Shock 13:267-273

15. Edouard AR, Degremont AC, Duranteau J et al (1994) Heterogeneous regional vascular responses to simulated transient hypovolemia in man. Intensive Care Med 20:414-420

16. Porta F, Takala J, Kolarova A et al (2005) Oxygen extraction in pigs subjected to low-dose infusion of endotoxin after major abdominal surgery. Acta Anaesthesiol Scand 49:627-634

17. Brandt S, Regueira T, Bracht $\mathrm{H}$ et al (2009) Effect of fluid resuscitation on mortality and organ function in experimental sepsis models. Crit Care 13:R186

18. Uusaro A, Lahtinen P, Parviainen I et al (2000) Gastric mucosal end-tidal $\mathrm{PCO}_{2}$ difference as a continuous indicator of splanchnic perfusion. Br J Anaesth 85:563-569

19. Bailey RW, Brengman ML, Fuh KC et al (2000) Hemodynamic pathogenesis of ischemic hepatic injury following cardiogenic shock/resuscitation. Shock 14:451-459

20. Tenhunen JJ, Uusaro A, Karja V et al (2003) Apparent heterogeneity of regional blood flow and metabolic changes within splanchnic tissues during experimental endotoxin shock. Anesth Analg 97:555-563

21. Riddez L, Hahn RG, Brismar B et al (1997) Central and regional hemodynamics during acute hypovolemia and volume substitution in volunteers. Crit Care Med 25:635-640

22. Jakob SM, Ruokonen E, Vuolteenaho O et al (2001) Splanchnic perfusion during hemodialysis: evidence for marginal tissue perfusion. Crit Care Med 29:1393-1398

23. Mackway-Jones K, Foex BA, Kirkman E et al (1999) Modification of the cardiovascular response to hemorrhage by somatic afferent nerve stimulation with special reference to gut and skeletal muscle blood flow. J Trauma 47:481-485

24. Foex BA, Kirkman E, Little RA (2004) Injury (nociceptive afferent nerve stimulation) modifies the hemodynamic and metabolic responses to hemorrhage in immature swine. Crit Care Med 32:740-746

25. Jakob SM, Kosonen P, Ruokonen E et al (1999) The Haldane effect-an alternative explanation for increasing gastric mucosal $\mathrm{PCO}_{2}$ gradients? Br J Anaesth 83:740-746

26. Lautt WW (1985) Mechanism and role of intrinsic regulation of hepatic arterial blood flow: hepatic arterial buffer response. Am J Physiol 249:G549-G556

27. Hiltebrand LB, Krejci V, tenHoevel ME et al (2003) Redistribution of microcirculatory blood flow within the intestinal wall during sepsis and general anesthesia. Anesthesiology 98:658-669

28. Brandstrup B, Tonnesen H, Beier-Holgersen R et al (2003) Effects of intravenous fluid restriction on postoperative complications: comparison of two perioperative fluid regimens: a randomized assessor-blinded multicenter trial. Ann Surg 238:641-648

29. Nisanevich V, Felsenstein I, Almogy G et al (2005) Effect of intraoperative fluid management on outcome after intraabdominal surgery. Anesthesiology 103:25-32 
30. McArdle GT, McAuley DF, McKinley A et al (2009) Preliminary results of a prospective randomized trial of restrictive versus standard fluid regime in elective open abdominal aortic aneurysm repair. Ann Surg 250:28-34

31. Arkilic CF, Taguchi A, Sharma N et al (2003) Supplemental perioperative fluid administration increases tissue oxygen pressure. Surgery 133:49-55

32. Holte K, Klarskov B, Christensen DS et al (2004) Liberal versus restrictive fluid administration to improve recovery after laparoscopic cholecystectomy: a randomized, double-blind study. Ann Surg 240:892-899

33. Shoemaker WC, Appel PL, Kram HB et al (1988) Prospective trial of supranormal values of survivors as therapeutic goals in high-risk surgical patients. Chest 94:1176-1186
34. Boyd O, Grounds RM, Bennett ED (1993) A randomized clinical trial of the effect of deliberate perioperative increase of oxygen delivery on mortality in high-risk surgical patients. JAMA 270:2699-2707

35. Wilson J, Woods I, Fawcett J et al (1999) Reducing the risk of major elective surgery: randomised controlled trial of preoperative optimisation of oxygen delivery. BMJ 318:1099-1103

36. Rivers E, Nguyen B, Havstad S et al (2001) Early goal-directed therapy in the treatment of severe sepsis and septic shock. N Engl J Med 345:1368-1377 